an extension of the original finding, which needs further replication itself to increase the likelihood that the reported association is real. Nevertheless, these studies, as well as failures to replicate, should be published to evolve a full picture of a gene's possible effects. Much can be learned from these findings and, in the end, they may be even more important for our understanding of genetics and disease mechanisms in complex diseases than straightforward replication.

Blakey et al studied five positionally cloned genes, initially found in linkage and association studies in children. They now aimed to replicate associations with asthma, wheezing and IgE in their large British population sample. They found small but significant effects for polymorphisms in DPP1O and ADAM33 (and borderline for GPR154), with odds ratios of around 1.1 per allele.

Is this the final verdict on the role of these genes in asthma? Rather not. Although this is a large and well phenotyped cross-sectional and probably unbiased population sample, it is a snapshot in time. The individuals studied were born in 1 week of 1958 in Britain. It may be assumed that environmental factors were very different at the time these individuals developed their asthma compared with children growing up nowadays. Looking back on the second half of the 20th century we have witnessed rising prevalence rates for asthma, and fundamental changes in lifestyle, diet, and personal and meta-environments. It could very well be that asthma of today's children and youth is the result of mechanisms different from those relevant for asthma development 50 years ago. However, when associations are found consistently across time, this could point to very basic and timeless asthma mechanisms. This is nothing but a hypothesis-but one to consider when indulging in replication studies such as this that look simple at first glance but may hold more food for thought than expected.

\section{Competing interests: None.}

Thorax 2009;64:370-371. doi:10.1136/thx.2008.105197

\section{REFERENCE}

1. Blakey JD, Sayers I, Ring SM, Strachan DP, Hall IP. Positionally cloned asthma susceptibility gene polymorphisms and disease risk in the British 1958 Birth Cohort. Thorax 2009;64:381-7.

\title{
Change in smoking status after low-dose spiral chest CT screening for lung cancer: opportunity for smoking intervention
}

\author{
Matthew M Clark, James R Jett ${ }^{2,3}$
}

Several studies have examined the effectiveness of low-dose spiral chest CT scan screening for early detection of lung cancer. Currently, most patients with lung cancer are diagnosed at an advanced stage. Early detection of lung cancer could help reduce the high mortality rate associated with lung cancer. It is hoped that the advanced technology of this new screening procedure may prove to be comparable to the reduction in mortality associated with having the recommended mammographies for early detection of breast cancer.

However, unlike most cancers, lung cancer is associated with a specific behaviour-namely, smoking cigarettes. It has been proposed that informing cigarette smokers of negative lung cancer screening

\footnotetext{
${ }^{1}$ Department of Psychiatry and Psychology, Mayo Clinic, Rochester, Minnesota, USA; ${ }^{2}$ Division of Pulmonary and Critical Care Medicine, Mayo Clinic, Rochester, Minnesota, USA; ${ }^{3}$ Division of Medical Oncology, Mayo Clinic, Rochester, Minnesota, USA

Correspondence to: Dr J R Jett, Division of Pulmonary and Critical Care Medicine, Mayo Clinic, Rochester, MN55905, USA; jett.james@mayo.edu
}

results could give them permission, licence or a "green light" to continue smoking. If a screening procedure leads to increased smoking rates, it is possible that any health benefits associated with early detection of lung cancer would be offset by an increase in the prevalence of lung cancer due to increased smoking rates.

Fortunately, several non-randomised screening programmes have examined this concern and, so far, negative screening results do not appear to increase smoking rates. For example, we found that CT screening results did not affect the smoking rates of 1475 adults with a smoking history of at least 20 pack-years at 1-year followup. ${ }^{1}$ Interestingly, in this same sample we found that three abnormal screens over a 3year period promoted smoking abstinence (41.9\%). Those with three negative screens still had a higher than expected quit rate $(19.8 \%)$ over the 3 years. ${ }^{2}$

The study by Ashraf and colleagues in this issue of Thorax (see page 388) is unique in that participants were randomised to receive either CT screening or to not have any screening. ${ }^{3}$ A randomised study design helps to further understand the possible effects that lung cancer screening may have on smoking rates. The fact that the authors also found that the screening and control subjects both demonstrated a quit rate of almost $12 \%$ is further support of previous research findings from single-arm studies that negative screening results do not promote or encourage continued cigarette smoking. The study also demonstrates the strength of a randomised controlled trial, and showed that smoking cessation was equal in the two arms regardless of whether or not the subjects received CT screening. Without the control arm it would be possible mistakenly to attribute the successful smoking cessation to having CT screening.

What is striking is that the quit rate found in participants in CT screeningeither in randomised trials or single-arm studies-is higher than expected. Participants usually have a 20 pack-year history of smoking so they are long-term addicted smokers. That almost $12 \%$ quit at 1 year in this study and that $14 \%$ quit at 1 year in our study shows that this is a population of smokers with a high motivation to quit smoking. Our attempt to take advantage of this teachable moment by enhancing smoking abstinence with either internet resources or written materials proved ineffective. ${ }^{4}$ It is our current opinion that more intensive, truly multidisciplinary approaches are needed for smoking cessation. We therefore suggest that smoking cessation interventions that combine pharmacotherapy with nicotine counselling and use screening results to enhance motivation for quitting smoking may prove to be efficacious for this 
population at high risk for lung cancer. Hopefully, future studies can design and implement efficacious tailored smoking cessation programmes for participants in lung cancer screening programmes. ${ }^{5}$

Competing interests: None.

Thorax 2009;64:371-372. doi:10.1136/thx.2008.111039

\section{REFERENCES}

1. Cox LS, Clark MM, Jett JR, et al. Change in smoking status after spiral chest computed tomography scan screening. Cancer 2003;98:2495-501.

2. Townsend CO, Clark MM, Jett JR, et al. Relation between smoking cessation and receiving results from three annual spiral chest computed tomography scans for lung carcinoma screening. Cancer 2005;103:2154-62.
3. Ashraf $\mathbf{H}$, Tonnesen $\mathrm{P}$, Pedersen $\mathrm{JH}$, et al. Effect of CT screening on smoking habits at 1-year follow-up in the Danish Lung Cancer Screening Trial (DLCST). Thorax 2009;64:388-92

4. Clark MM, Cox LS, Jet JR, et al. Effectiveness of smoking cessation self-help materials in a lung cancer screening population. Lung Cancer 2004;44:13-21.

5. Taylor KL, Cox LS, Zincke N, et al. Lung cancer screening as a teachable moment for smoking cessation. Lung Cancer 2007:56:125-34.

\section{Sex, cough and (digital) audiotape}

\section{Peter V Dicpinigaitis}

Cough is among the most common complaints for which medical attention is sought ${ }^{1}$ yet, historically, despite its significance as a health issue worldwide, cough has been under-represented in terms of basic and clinical research efforts aimed at understanding its mechanisms and developing effective therapeutic agents. Thankfully, the past decade has witnessed an emergence of interest in cough within the scientific community. Highlighting the perceived importance of cough as a clinical problem, no less than five major pulmonary societies have published guidelines addressing the management of cough: the European Respiratory Society, ${ }^{2}$ the German Respiratory Society, ${ }^{3}$ the American College of Chest Physicians, ${ }^{4}$ the British Thoracic Society $^{5}$ and the Japanese Respiratory Society. ${ }^{6}$ In addition, a task force of the European Respiratory Society published the first ever guidelines on the assessment of cough, ${ }^{7}$ aimed at promoting and improving the quality of cough-related research. Furthermore, the discovery of several pharmacological receptors relevant to the cough reflex-such as TRPV $1,{ }^{8} \mathrm{NOP} 1^{9}$ and cannabinoid receptors ${ }^{10}$ — has stimulated numerous investigative programmes within the pharmaceutical industry.

Research efforts in cough continue to be hindered by a dearth of clinically relevant, well validated measurement tools. Although cough reflex sensitivity can be accurately and reproducibly determined using inhalational (capsaicin or citric acid) cough challenge methodology, results generated in the laboratory may not correlate with the clinical state. ${ }^{11}$ For example, a potential antitussive agent under investigation may demonstrate the

Correspondence to: Dr P V Dicpinigaitis, Albert Einstein College of Medicine, 1825 Eastchester Road, Bronx, NY10461, USA; pdicpinigaitis@pol.net ability to inhibit experimentally-induced cough but fail to suppress pathological cough. ${ }^{11}$

Subjective cough-specific instruments such as the Leicester Cough Questionnaire $(\mathrm{LCQ})^{12}$ and the Cough-specific Quality of Life Questionnaire (COLO) ${ }^{13}$ have been published, validated and have served as useful tools in clinical cough research. However, factors such as mood, level of vigilance, symptoms associated with cough (pain, vomiting, incontinence, syncope) and placebo effect ${ }^{14}$ may influence an individual's perception of cough severity and frequency, as well as the effect of a pharmacological intervention on these parameters. Indeed, studies have demonstrated that subjective measures of cough are only moderately related to objectively measured cough. ${ }^{15}$ Thus, the optimal use of subjective symptom assessment tools would appear to be in conjunction with objective cough measurement. The ideal objective cough monitor would be a portable, ambulatory, digital-recording device able to store at least $24 \mathrm{~h}$ of information that could then be analysed using software capable of recognising a cough with adequate sensitivity and specificity. Although several objective cough monitoring systems are currently under development, only one is commercially available at present. ${ }^{16}$

In this issue of Thorax, Kelsall and colleagues $^{17}$ employ their objective cough monitoring system (Vitalojak, Vitalograph, UK) to investigate whether, among patients with chronic cough presenting to a tertiary referral clinic, women have higher cough rates than men (see page 393). Furthermore, they examined whether other predictors of objective cough frequency could be discerned.

It is not surprising that women were shown to have significantly higher cough rates, albeit only during the night. Women are consistently over-represented in specialty referral centres. Previous studies have shown that healthy women have a more sensitive cough reflex than that of healthy men. ${ }^{18} 19$ The present study confirms the results of a previous trial of patients with pathological cough, in which women had heightened cough sensitivity compared with men. ${ }^{20}$ Notably, though, in this study sex and cough reflex sensitivity (to citric acid) independently predicted higher cough rates, implying that factors other than cough reflex sensitivity (and still to be identified) may be contributing to a greater amount of coughing in women. The inability of the LCQ to demonstrate a significantly poorer cough-related quality of life in women, despite a much higher cough rate than men, reminds us that a particular subjective instrument may omit information relevant to a particular study population. The ability of the COLO to discern sex-related differences ${ }^{21} 22$ probaby reflects its inclusion of items particularly distressful to women with chronic cough, such as urinary incontinence.

Remarkable in this study is the finding that older subjects had higher cough frequency than younger subjects. There appears to be a presumption in the literature that cough reflex sensitivity is diminished in elderly subjects because patients with a history of aspiration pneumonia, most of whom are elderly, have been shown to have a diminished response to citric acid. ${ }^{23}$ One study specifically evaluated cough reflex sensitivity to citric acid in a group of 110 subjects (60 men; age range 20-78 years) divided into groups from the third to eighth age decades. Cough reflex sensitivity did not differ significantly among the six age decades, suggesting that cough reflex sensitivity does not diminish with age. ${ }^{25}$ Regardless, the authors of the present study found that the relationship between cough frequency and age was independent of cough reflex sensitivity as well as duration of cough. Thus, an explanation for the increased cough frequency in older subjects in this study remains elusive (gastro-oesophageal reflux?), but provides fertile ground for further research. 